\title{
Numerical modelling of optical trapping in hollow photonic crystal cavities
}

\author{
Ulagalandha Perumal Dharanipathy • Romuald Houdré
}

Received: 30 September 2011 / Accepted: 17 January 2012 / Published online: 5 February 2012

C) Springer Science+Business Media, LLC. 2012

\begin{abstract}
Photonic crystal (PhC) devices owing to their strong confinement of electromagnetic energy are considered to be excellent candidates for on chip optical trapping of dielectric or biological particles in the nanometer range. In this work, we study and present hollow PhC cavities and characterize them for their trapping stiffness, trapping stability and variation of resonance wavelength due to the presence of various sized single particles in the cavity.
\end{abstract}

Keywords Optical trapping - Single particle detection · Photonic crystal cavities . Finite element methods

\section{Introduction}

Photonic crystals (PhCs) are periodic nanostructures well known for their confinement properties of light (Yablonovitch 1987). Light confined in smaller dimensions can be very useful for applications that require gradients of electromagnetic field in sub-wavelength spatial dimensions (Song et al. 2005). Optical trapping of particles is one such application where gradient fields are used to trap particles with non-resonant laser beams since the 1970s. PhC cavities, with their field confinement and the resulting gradients are promising candidates for on-chip optical trapping studies. In general, cavities formed in $\mathrm{PhCs}$ confine light in the medium with higher refractive index region. For applications such as optical trapping, it would be beneficial to tailor the cavity modes such that the light is more confined in the lower dielectric (Almeida et al. 2004).

A hollow PhC cavity is formed when maximum field is concentrated in the air when a defect is created in the PhC slab. These cavities are more suitable for trapping as they have a maximum field overlap with lower dielectric and moreover they provide us with geometric confinement in the in-plane direction and a possibility of a strong gradient in the vertical

U. P. Dharanipathy $(\varangle) \cdot$ R. Houdré

Laboratory of Quantum Optoelectronics, Ecole Polytechnique Fédérale de Lausanne (EPFL), Station 3,

1015 Lausanne, Switzerland

e-mail: ulagalandha.dharanipathy@epfl.ch 

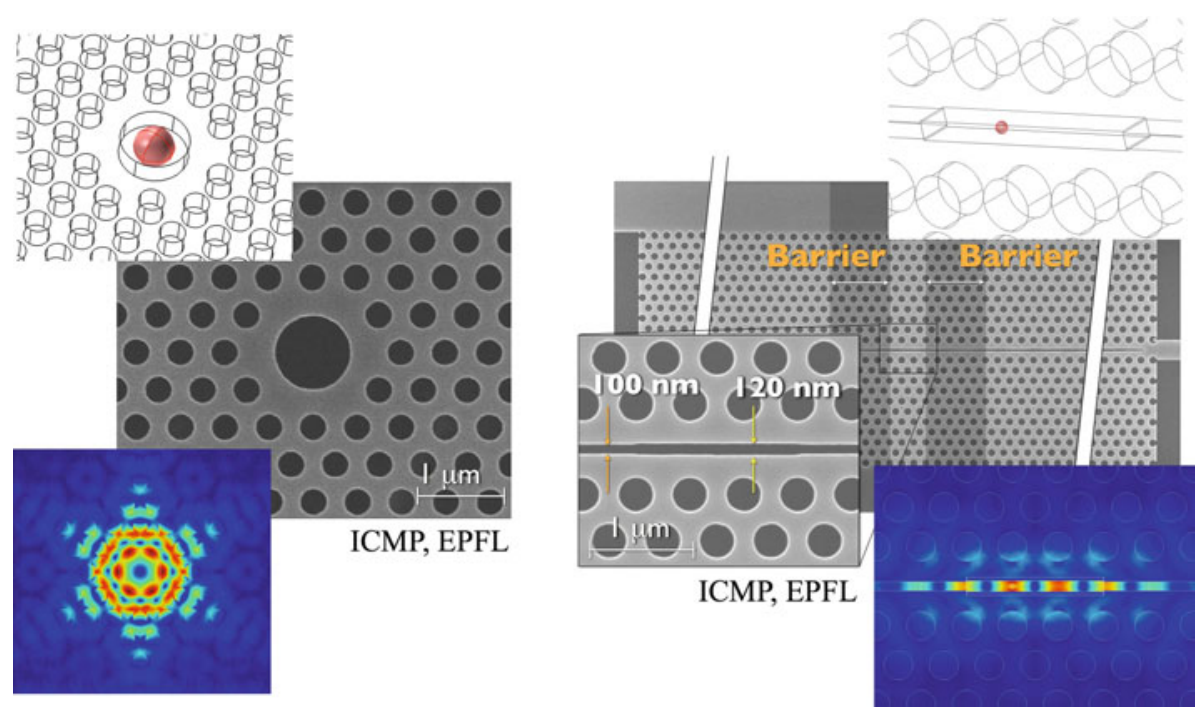

Fig. 1 Hollow PhC cavities: SEM image (middle), electric field norm (bottom inset), simulation of particle in the defect (top inset) (Left: holey defect, right: slot defect)

direction as well. Figure 1 shows the two hollow $\mathrm{PhC}$ devices that are studied in detail in this paper, the holey defect (Lee and Fauchet 2007) where the central hole is three times larger than the surrounding holes giving rise to multiple defect modes in the band-gap and the slot defect (Jágerská et al. 2010) where the slot mode is confined in the center with the help of barrier regions formed between the two differently sized slots.

\section{Metrics in a generic PhC cavity for optical trapping}

In consideration to optical trapping, we assign four physical metrics pertaining to a cavity mode.

The quality factor (Q) of a cavity gives a figure of merit for the radiation and absorption losses present in the system. A sufficient $Q$ in the infiltrated medium of interest is necessary in order to have field confinement required for trapping a particle.

$$
\mathrm{Q}_{\text {factor }}=\frac{2 \pi f_{0}(\text { Energy Stored })}{(\text { Energylost per cycle })}
$$

The effective mode volume $\left(\mathrm{V}_{\text {eff }}\right)$ is used to determine the active sensing volume and the sensitivity of the cavity mode, which in turn influences the forces exerted by the field on the particle.

$$
\mathrm{V}_{\mathrm{eff}}=\frac{\iiint\left(f(r) d^{3} r\right)^{2}}{\iiint f^{2}(r) d^{3} r} \text { where } f(r)=\varepsilon(r)|\mathrm{E}(r)|^{2}
$$

The cavity mode eigen energy responds to the changes in the real part of the refractive index of the surrounding medium. This corresponds to a change in the resonance wavelength of the cavity mode. In the presence of a Brownian motion of a dielectric particle in the environment, this effect is dynamic and could also be used to track the movement of the 


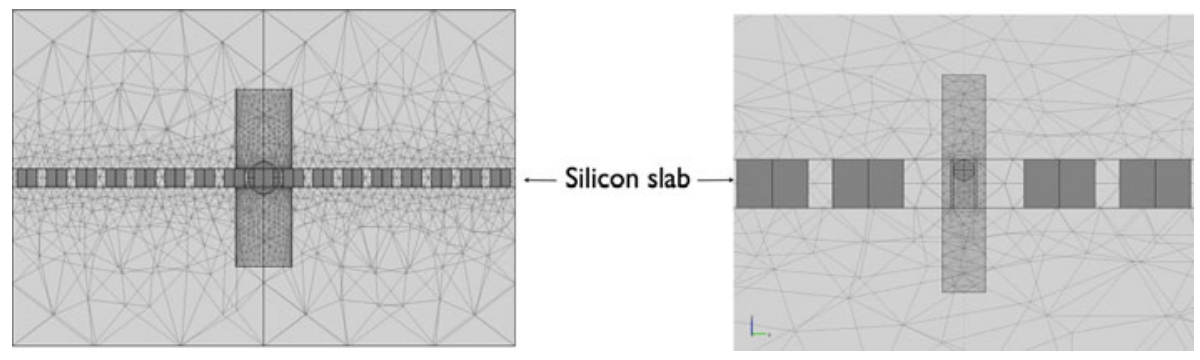

Fig. 2 Meshing of geometries (vertical cross section). holey cavity (left) and slot cavity (right) with $2 \mu \mathrm{m}$ air on top and bottom

particle and facilitate resonant trapping by back action. This resonant trapping of the cavity mode assisted by back action is in contrast to the conventional optical trap wherein the particle has no influence on the frequency of the trapping field.

$$
\frac{\Delta \lambda}{\Delta n}=\Gamma \frac{\lambda}{n_{\mathrm{eff}}}
$$

The trap potential is a complex parameter that is related to the Q factor of the cavity, the power that is injected into the cavity, the difference in the permittivity of the surrounding medium and the stiffness ( $\kappa$ trap) resulting due to the gradient fields arising in the cavity mode. The particle is trapped for longer or shorter times depending on the depth of the potential well.

$$
\mathrm{U}_{\text {trap }} \alpha \mathrm{Q}_{\text {cavity }}, \mathrm{P}_{\text {cavity }}, \Delta n, \kappa_{\text {trap }} \text { and } \mathrm{F}=-\kappa_{\text {trap }} x
$$

\section{Modelling methodology}

The hollow cavity configurations fit well within the requirements of the above described metrics and hence we numerically analyse these cavities for on-chip optical trapping with 3D finite element methods. The particles used were dielectric particles. Various size and position dependent studies were used to compute the variation of resonance frequencies and the gradient forces acting on the particle. The model included a $220 \mathrm{~nm}$ silicon (index of 2.46) $\mathrm{PhC}$ slab with cavities and $2 \mu \mathrm{m}$ of water above and below the slab as shown in Fig. 2 . The surrounding medium and the holes are filled with water with refractive index 1.35. The $\mathrm{PhC}$ holes, the cavity and the particle were meshed adaptively and a constant meshing cylinder was placed around the particle as shown in Fig. 2 to ensure mesh convergence in the solution.

First order absorbing boundary conditions were enforced on all the exterior boundaries and continuity condition within the insides of the domains.

\section{Numerical results}

\subsection{Q factors}

The Q factors are computed with the real part and imaginary part of the eigenfrequency in finite element methods (Table 1). 
Table 1 Calculated and experimental Q factors of hollow cavities

\begin{tabular}{|c|c|c|c|c|}
\hline & \multicolumn{2}{|c|}{ Holey cavity } & \multicolumn{2}{|l|}{ Slot cavity } \\
\hline & Calculated & Experiment & Calculated & Experiment \\
\hline Q air & 7,630 & $\sim 4,000$ & 28,000 & $\sim 26,000$ \\
\hline Q water & 3,477 & $\sim 1,000$ & 5,500 & - \\
\hline
\end{tabular}
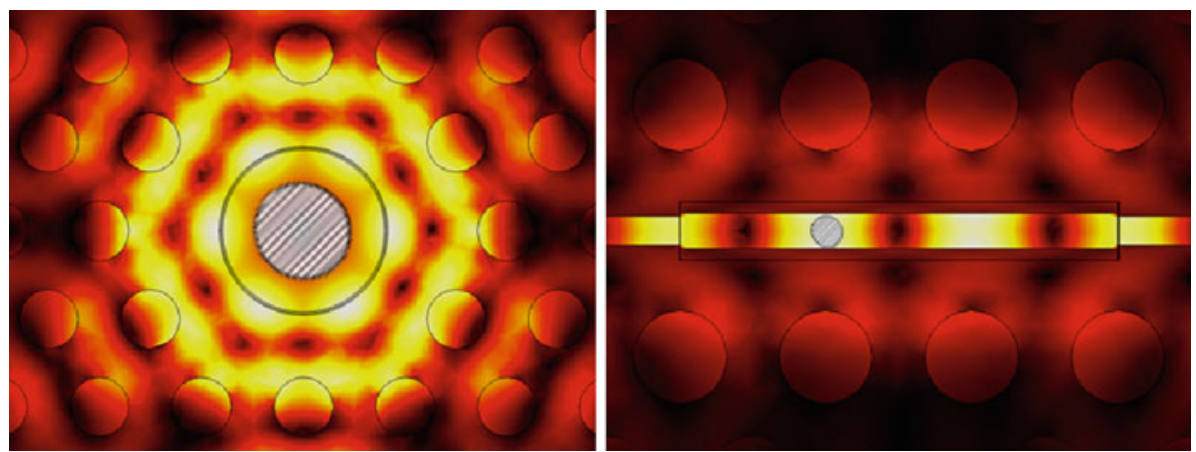

Fig. 3 In plane cross section of field overlaps on particle (striped circle) (left: holey cavity with a $400 \mathrm{~nm}$ particle in the centre, Right: slot cavity with a $100 \mathrm{~nm}$ particle in the centre of the left lobe)

The Q factors in the presence and absence of water on the holey defect $\mathrm{PhC}$ cavity has been verified experimentally by bulk infiltration experiments and they are in agreement with the numerical results.

\subsection{Spatial extents and overlap of field}

The spatial extent of the electric field over the particle determines the strength of the trap.

The larger the overlap, the larger the gradient forces acting on the particle. The hexapole field configuration shown for the holey defect cavity makes it very size specific as larger particles 'see' the influence of the field better than the particles that are smaller than $100 \mathrm{~nm}$. The holey defect is $700 \mathrm{~nm}$ in diameter and we are seeing an exponential drop of the fields from the edge of the cavity to the center and hence the particle experiences this field from the edges of the holey defect, provided the particle is of a larger diameter. The decay of the field in the vertical direction has its significance as it has to allow the particle to enter the hollow cavity region. The decay length of the field has been found to be around $250 \mathrm{~nm}$ from the top surface of the silicon slab and if a particle flowing by in this region experiences a force towards the cavity center, the particle is pulled inside the hollow cavity region (Fig. 3).

\subsection{Resonance frequency shift}

The continuous motion of the particle in the vicinity of the cavity causes a dynamic shift of the cavity resonance wavelength due to the shift of the mode in the dispersion of the $\mathrm{PhC}$. Particles of different sizes are placed in the center of the cavity region. They are displaced discretely along the vertical direction until the particle is sufficiently far from the surface of the silicon slab. The resonance frequency is then calculated at every position.

In Fig. 4, it is shown that the particle causes maximum shift until it is inside the silicon slab region and then the shift drastically drops as the particle moves farther and farther away from 

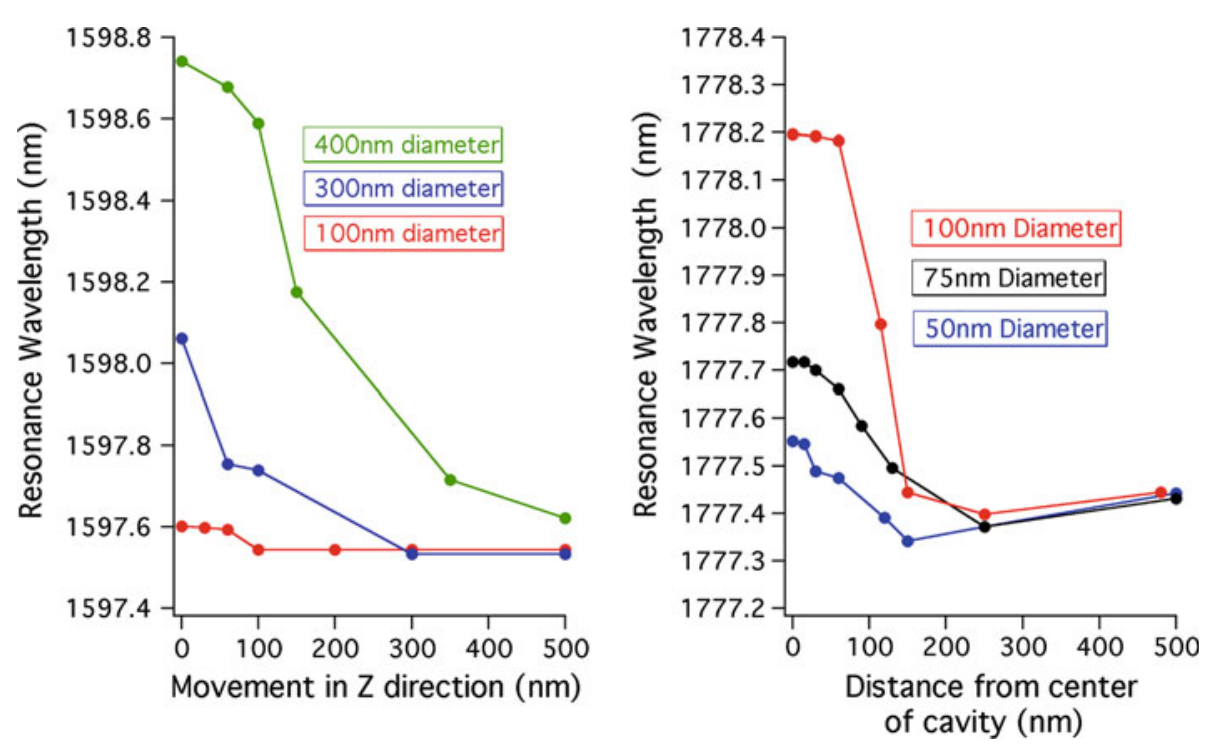

Fig. 4 Resonance frequency shift against movement of the particle in the vertical direction placed inside the cavity region (left: holey cavity, right: slot cavity)

the silicon slab, which corresponds to the decay length explained before. The effect is more prominent for the larger particles for two main reasons, primarily due the difference in the refractive index of the surrounding medium and secondly due to the interaction of the field on the larger particles. The values of the resonance shift are of the order of the linewidth of the cavity mode and so they could be experimentally verified and the tracking of the resonance wavelength could be used for dynamic tracking of the motion of the particle. It could also be used for a single particle detection scheme with the amount of resonance shift induced by the particle.

\subsection{Trapping forces and stiffness}

The resonant frequency of the cavity field shifts due to the presence of the particle and so there is a constant buildup of field as the particle moves inside the volume. We perform a stationary analysis by placing the particle inside the cavity volume in the center of the cavity and then we parametrically move the particle towards the exterior of the silicon slab in the vertical direction. The force experienced by the particle as shown in Fig. 5 is then computed by integrating the surface of the particle using the Maxwell Stress Tensor (MST) approach (Wang et al. 1997).

The total energy stored in the cavity is computed by integrating the energy density within volume of the cavity with 1 watt input power. The power dissipation in watts can then be computed with the ratio of energy stored in the cavity to the $\mathrm{Q}$ factor of the cavity in water. This value is used for normalizing all the computed forces and potentials to 1 watt of power. These forces when plotted against the distance in vertical direction produce a force-distance curve where the maximum force is seen around the edge of the silicon slab and the force decreases exponentially afterwards in Fig. 5. The slope of the curve inside the slab region can be used to calculate the maximum stiffness experienced by the particle over this distance. The force experienced by a $100 \mathrm{~nm}$ particle in the case of the holey cavity is very small given 

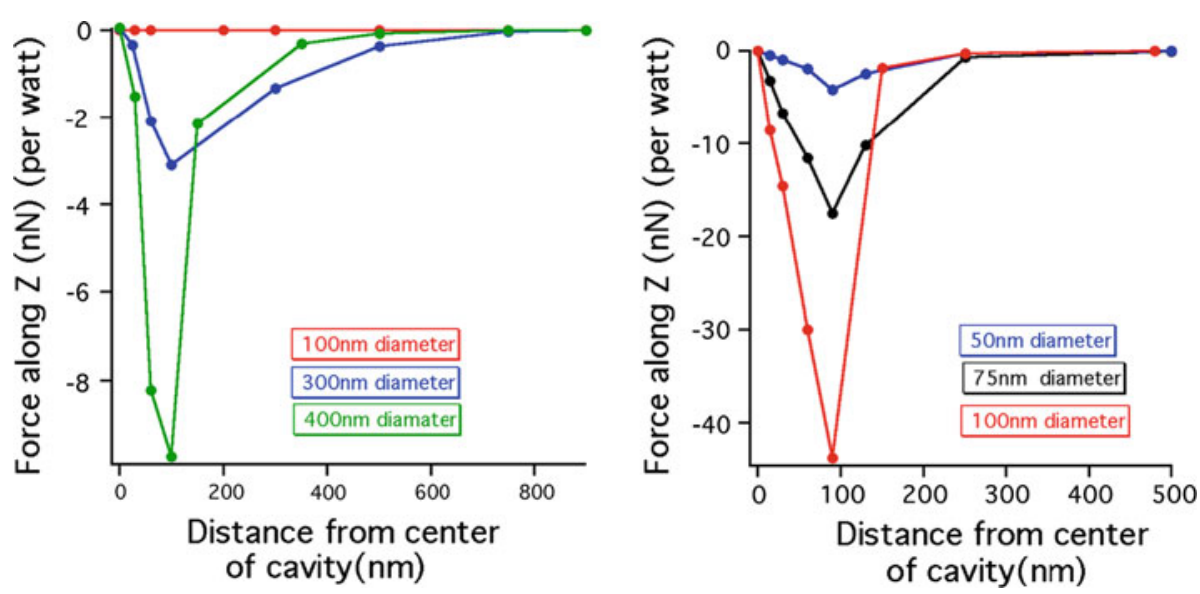

Fig. 5 Force-distance curve for the motion of the particle in the vertical direction placed inside the cavity region (left: holey cavity, right: slot cavity)
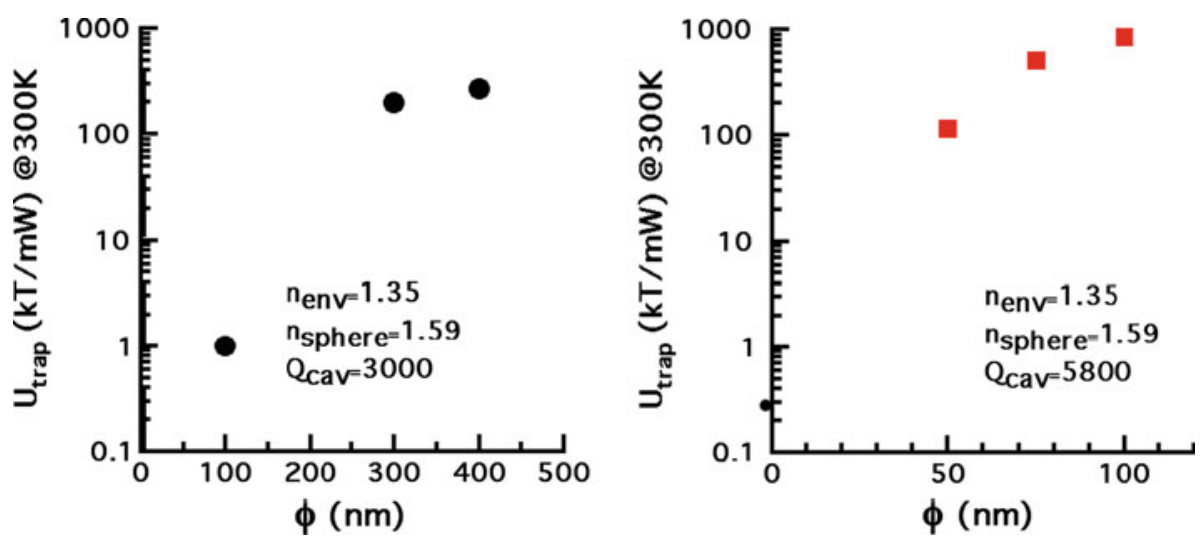

Fig. 6 Trapping potentials versus diameter of particles (left: holey cavity, right: slot cavity)

the fact that the particle is placed in the radial center of the holey defect. It has been verified that the forces are scaled up when the particle is positioned close to the edges of the hole in its horizontal plane where the particle experiences a larger influence of the field.

\subsection{Trap potential}

The trapping potential denotes the depth of the potential well due to the gradient fields in the cavity region. The trapping potential is then computed by integrating the force distance curve from Fig. 6 . The potentials are plotted against the diameter of dielectric particles $(n=1.59)$ placed in the cavity region. The trapping potential is the minimum energy required by the particle to escape the optical trap. A higher trapping potential could also result in extended trapping times for overcoming the Brownian motion of the particle. As it is shown in Fig. 6 , the trapping potential is higher for the slot type defect owing primarily to its acute field confinement and a larger mode overlap. 
Table 2 Comparison of stiffness and maximum forces for particles of different sizes

\begin{tabular}{lllllll}
\hline \multicolumn{2}{l}{ Holey cavity } & & \multicolumn{2}{l}{ Slot cavity } \\
\cline { 1 - 3 } $\begin{array}{l}\text { Size } \\
(\mathrm{nm})\end{array}$ & $\begin{array}{l}\text { Maximum force } \\
(/ \mathrm{W})\end{array}$ & $\begin{array}{l}\text { Stiffness 'k' in 'z' } \\
(\mathrm{pN} / \mathrm{nm} / \mathrm{W})\end{array}$ & & $\begin{array}{l}\text { Size } \\
(\mathrm{nm})\end{array}$ & $\begin{array}{l}\text { Maximum force } \\
(/ \mathrm{W})\end{array}$ & $\begin{array}{l}\text { Stiffness 'k' in 'z' } \\
(\mathrm{pN} / \mathrm{nm} / \mathrm{W})\end{array}$ \\
\hline 100 & $13 \mathrm{pN}$ & 0.12 & 50 & $4.2 \mathrm{nN}$ & 46.2 \\
300 & $3.1 \mathrm{nN}$ & 28.1 & 75 & $17.5 \mathrm{nN}$ & 195 \\
400 & $9.7 \mathrm{nN}$ & 88.5 & 100 & $45.6 \mathrm{nN}$ & 506.9 \\
\hline
\end{tabular}

\section{Comparison of trapping metrics}

The holey cavity with its large size is capable of holding and trapping particles of diameter up to $500 \mathrm{~nm}$ in size, which could correspond to large dielectric particles or bacteria. The slot cavity on the other hand is able to handle particles of the order of $50 \mathrm{~nm}$, which could correspond to proteins or small nanoparticles. The forces experienced by the small particles in the slot are relatively larger compared to the holey cavity because of the field configuration inside the cavity volume. The computed theoretical values are definitely larger in terms of maximum forces and stiffness in $z$-axis in comparison to standard conventional optical tweezers and other resonant trapping schemes as listed here (Serey et al. 2010) (Table 2).

\section{Conclusion}

We have demonstrated the application of $\mathrm{PhC}$ cavities, specifically the hollow $\mathrm{PhC}$ cavities for potential optical trapping studies. We have established figure of merits for a generic $\mathrm{PhC}$ cavity that could be used in trapping. Two different types of hollow cavities were studied with respect to the trapping metrics and we have computed the resonance frequency shifts, $\mathrm{Q}$ factor changes and trapping potentials against size and position dependence of particles in liquid media.

\section{References}

Almeida, V.R., Xu, Q., Barrios, C.A., Lipson, M.: Guiding and confining light in void nanostructure. Opt. Lett. 29, 1209-1211 (2004)

Jágerská, J., Zhang, H., Diao, Z., Le Thomas, N., Houdré, R.: Refractive index sensing with an air-slot photonic crystal nanocavity. Opt. Lett. 35, 2523-2525 (2010)

Lee, M.R., Fauchet, P.M.: Nanoscale microcavity sensor for single particle detection. Opt. Lett. 32, 32843286 (2007)

Song, B., Noda, S., Asano, T., Akahane, Y.: Ultra-high-Q photonic double-heterostructure nanocavity. Nature Mater 4, 207-210 (2005)

Serey, X., Mandal, S., Erickson, D.: Comparison of silicon photonic crystal resonator designs for optical trapping of nanomaterials. Nanotechnology 21, 305202 (2010)

Wang, X., Wang, X.-B., Gascoyne, P.R.C.: General expressions for dielectrophoretic force and electrorotational torque derived using the Maxwell stress tensor method. J. Electrostat. 39(4), 277-295 (1997)

Yablonovitch, E.: Inhibited spontaneous emission in solid-state physics and electronics. Phys. Rev. Lett. 58, 2059-2062 (1987) 